\title{
Pre-peritoneal prosthetic herniorraphy
}

\author{
LAURENCE F. TINCKLER \\ M.D., Ch.M., F.R.C.S., F.A.C.S., D.T.M. \& H. \\ Surgeon, Royal Gwent Hospital, Newport, Monmouth
}

\begin{abstract}
Summary
The usual operations for inguinal hernia suffer from a number of undesirable features, which are briefly discussed.

A new operation is described, in which the sites for potential herniation are protected with a synthetic prosthesis, as in Usher's method of incisional herniorrhaphy (1962).
\end{abstract}

MuLTIPLICITY of treatments for a condition implies that none is entirely satisfactory. This is true so far as operations for inguinal hernia are concerned and consequently the quest for the ideal operation continues.

Operations for inguinal hernia, as they are most usually performed, have in my opinion several undesirable features which are as follows:

The inguinal canal is opened up and its contents disturbed: in mobilizing the hernial sac the blood supply of the testes may be damaged leading to testicular atrophy, or the ilio-inguinal nerve traumatized giving rise to groin neuralgia; a repair approximating the conjoint tendon to Poupart's ligament or Gimbernat's ligament distorts tissues out of their normal anatomical alignment, attempts to unite muscle to ligament possibly under tension and utilizes tissues which have demonstrated their incompetence by the very presence of the hernia. All these features on general principles would be best avoided if acceptable alternative methods are available and I submit that the operation herein described attains this object.

It consists essentially of a pre-peritoneal approach to the deep aspect of the inguino-femoral area and the insertion of a synthetic fabric prosthesis to cover the potential sites of inguinal and femoral hernia. It has been evolved from the following reasoning.

The first layer of the abdominal parietes which is transgressed in the development of a hernia is the transversalis fascia; it would seem logical, therefore, to reinforce this layer in order to prevent recurrence.

Hernia repairs based on reinforcing the posterior wall of the inguinal canal at the level of the conjoint tendon savour of "closing the stable door after the horse has bolted'. In my experience of the hernia operation described by Nyhus \& Harkins (1964) which entails a suture repair of the transversalis fascia via a pre-peritoneal approach, this layer has mostly appeared to me to be insubstantial and I have had no confidence in relying upon it to effect a repair, despite the contention of Moschcowitz (1915) that 'It is an established truth that the transversalis fascia as a rule is strong enough to retain the viscus within the abdomen'.

The situation appears to call for the insertion of a prosthesis to reinforce the deepest layer of the abdominal parietes in the prevention of a recurrent hernia, either inguinal or femoral, and accordingly I have taken to reinforcing the fascia transversalis with Marlex mesh (Usher, 1963).

Marlex is a mono-filamentous mesh of high density polyethylene. It has high tensile strength, provokes very little foreign body reaction and is well tolerated in animal tissues; infection studies in dogs have shown that granulation tissue will grow through the mesh in the presence of purulent infection without slough or sinus formation. These are physical and biological properties favourable to the use of Marlex as a prosthesis in the repair of hernia and for the support of defective tissue (Usher, Oschner \& Tuttle, 1958).

When evaluated by animal experiment a satisfactory growth of fibrous tissue, through the mesh, was found to take place in about 6 weeks following implantation of the prosthesis. This layer of fibrous tissue was found to be quite uniform, pliable, and about 4-5 $\mathrm{mm}$ thick; there was no increase in the amount of fibrous tissue replacement after 6 weeks (Usher, 1959).

In a large series of incisional herniorrhaphies using Marlex mesh Usher (1962) encountered sterile seromas in $6 \%$, wound infections in $6 \%$ and persistent sinuses in 3\%: this last complication has not occurred since he changed from using silk or cotton to braided Marlex sutures for fixing the mesh in position; on occasions when it was necessary to open wound abscesses widely granulation tissue was observed growing through the mesh with subsequent complete wound healing by secondary intention. 
The steps of the operation, which were worked out by preliminary studies on autopsy material, are as follows:

\section{Anaesthesia}

Full relaxation of the abdominal wall is obtained by the use of muscle relaxants or alternatively spinal anaesthesia.

\section{Bladder}

In order to ensure that the bladder does not impede the pre-peritoneal exposure, the patient voids urine immediately before operation or alternatively, if the bladder is found to be inconveniently full at operation, it can be emptied by inserting a large bore needle through the bladder wall and applying suction.

\section{Incision and approach}

A mid-line supra-pubic incision from the umbilicus to the symphasis pubis is carried out, and the deep aspects of the inguino-femoral region approached

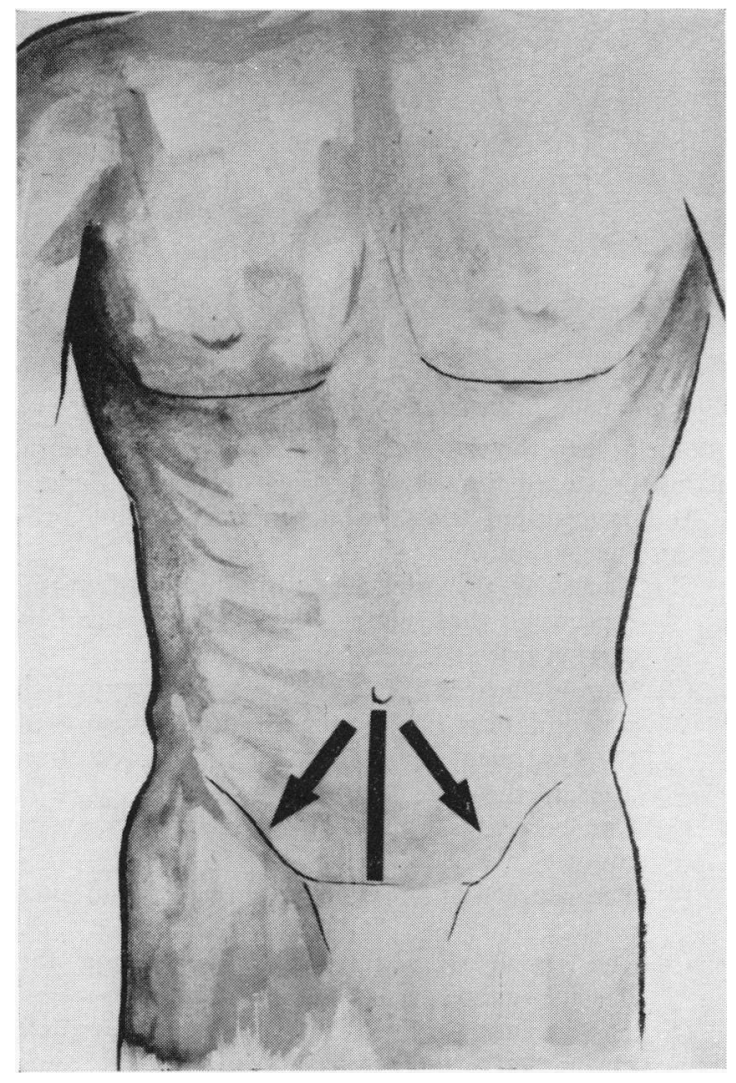

Fig. 1. A mid-line infra-umbilical incision gives access to both inguino-femoral regions. extraperitoneally, displacing the bladder backwards (Henry, 1963). A good view of the deep aspects of the inguino-femoral regions on both sides and the hernial sac at the site of its junction with the parietal peritoneum is obtained (Fig. 1).

\section{The hernial contents}

The hernial sac is explored by opening the parietal peritoneum and reducing any intraperitoneal contents, which may have entered the sac, by traction.

Should contents of a hernial sac be found irreducible owing to adhesions within the sac it is necessary to make a counter incision over the inguinal canal, expose and open the sac within the canal, but without the necessity of mobilizing it, and liberate the contents by dividing the adhesions. The further conduct of the operation is then carried out via the preperitoneal approach.

Again a hernia en glissade may be amenable to being reduced en masse by traction from the preperitoneal aspect, if not it is necessary to mobilize the sac within the inguinal canal via a separate incision to enable the hernial mass to be drawn back into the abdominal cavity.

\section{The hernial sac}

The hernial sac may be dealt with in one of two ways, either by drawing it back into the abdominal cavity by traction or alternatively circumcizing the sac at its neck, closing the resultant defect in the parietal peritoneum with a running catgut suture and leaving the fundus of the sac in situ with its proximal cut end left open. By dealing with the sac in this manner the contents and structure of the inguinal canal are left undisturbed.

\section{The hernial repair}

A double layer of Marlex mesh cut to appropriate size is now prepared. Sutures of Marlex material, left long and temporarily held by the application of haemostats, are placed in the following sites: one in the fascia overlying the pubic tubercle, two sutures in the fascia overlying the superior pubic ramus (Cooper's fascia) and one or two sutures in the iliopubic tract lateral to the internal inguinal ring (Fig. 2). The ilio-pubic tract is a condensation of the transversalis fascia and is best identified by palpation as a taut cord. Identified in this manner, it is grasped with tissue forceps and thus steadied for the insertion of the Marlex stitch. This latter stitch should be inserted by striking the needle from below upwards to avoid injuring the subjacent femoral vessels. These sutures are reloaded on needles and passed in mattress fashion through the lower border of the Marlex mesh (Fig. 3). The mesh is now manipulated down into position along the sutures which are then 


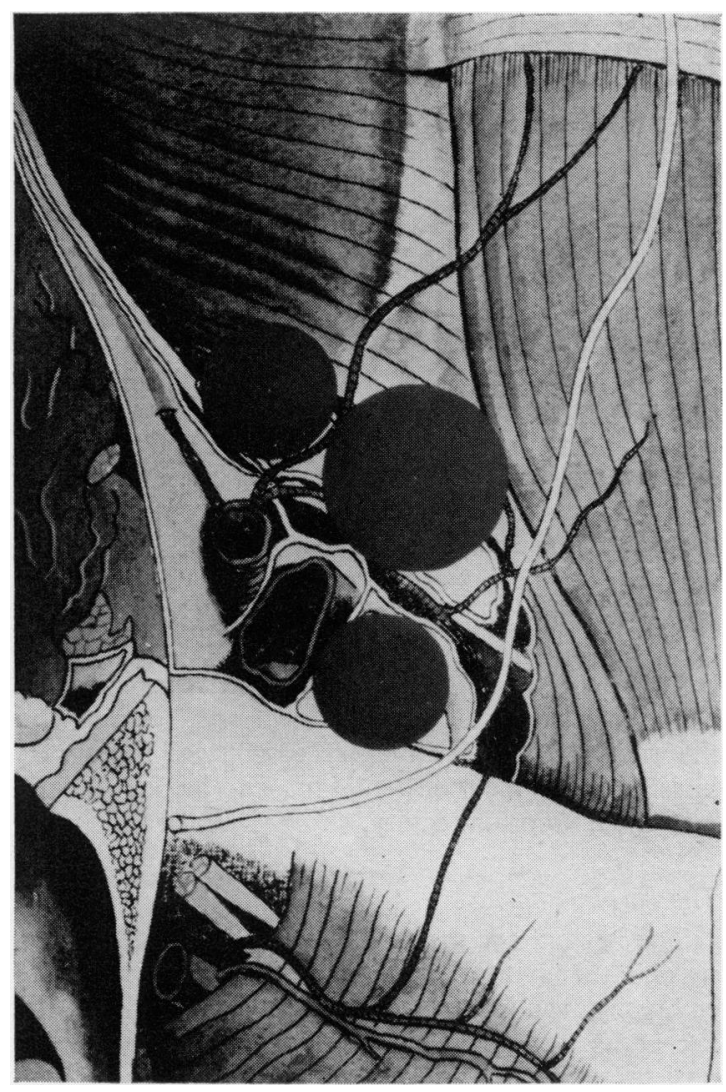

FIG. 2. The deep aspect of the left inguino-femoral area as seen at operation from the mid-line incision. Site of direct and indirect inguinal and femoral hernia are indicated in the diagram by discs.

tied serially (Fig. 4). The remaining margins of the Marlex prosthesis are sutured in position to the posterior aspect of the rectus muscle by interrupted Marlex sutures. If there is any tendency of the lower border of the Marlex prosthesis to compress the femoral vessels or the spermatic cord such compression can be readily relieved by suitably slitting the mesh. Having been placed in position the prosthesis effectively buttresses the femoral canal, the triangle of Hesselbach and controls the internal inguinal ring.

\section{Closure}

The mid-line wound is closed with interrupted catgut sutures. The wound is usually dry enough to justify closure without drainage; however, if there is any tendency to oozing, a drain may be inserted and suction applied for $24 \mathrm{hr}$.

\section{Antibiotics}

As the procedure entails insertion of foreign

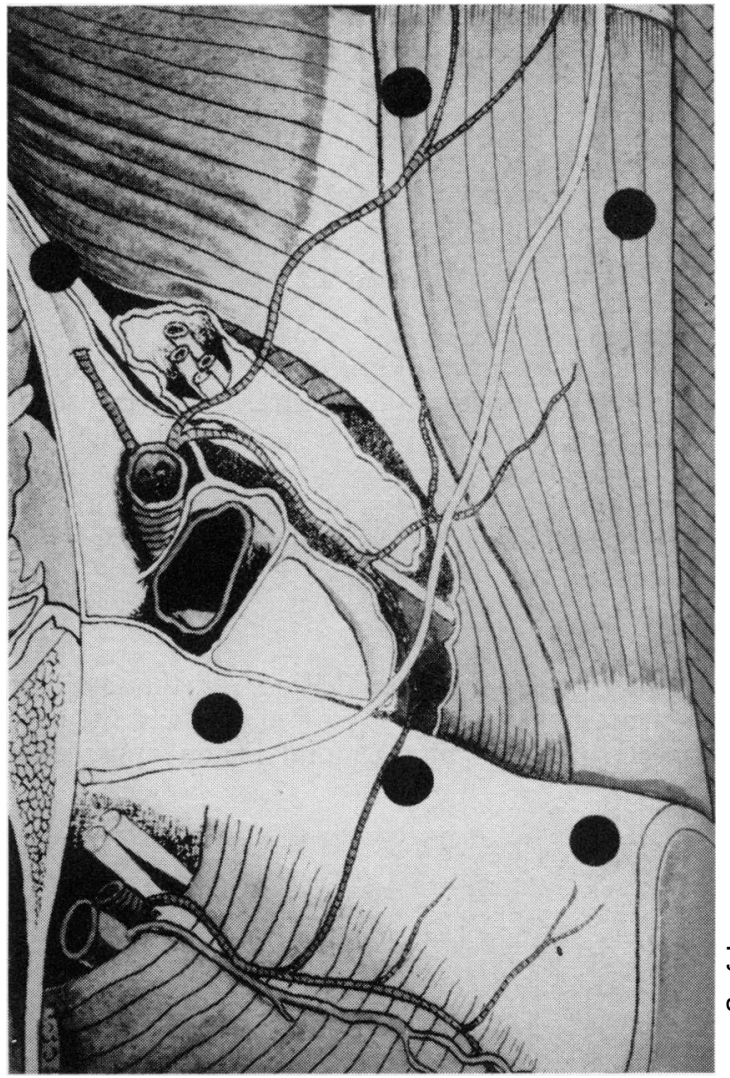

FIg. 3. Sites at which Marlex sutures are inserted to hold the Marlex mesh in position are indicated by black dots.

material into the tissues a broad spectrum antibiotic is administered for the first 5 post-operative days.

It is submitted that the advantages of this procedure are as follows:

(1) Access is provided to both inguino-femoral regions so that bilateral hernia repairs can be carried out at the same operation.

(2) Should bowel resection be necessary when strangulation has occurred, full exposure of the peritoneal cavity is available and resection and anastomosis facilitated.

(3) The hernial sac is dealt with directly at its source.

(4) The repair controls all potential hernial sites in that area.

(5) The repair does not rely on utilizing the possibly effete tissues of the patient.

(6) The inguinal canal and its contents are left untouched.

(7) The normal anatomy of the inguinal canal is preserved and the repair entails no distortion 


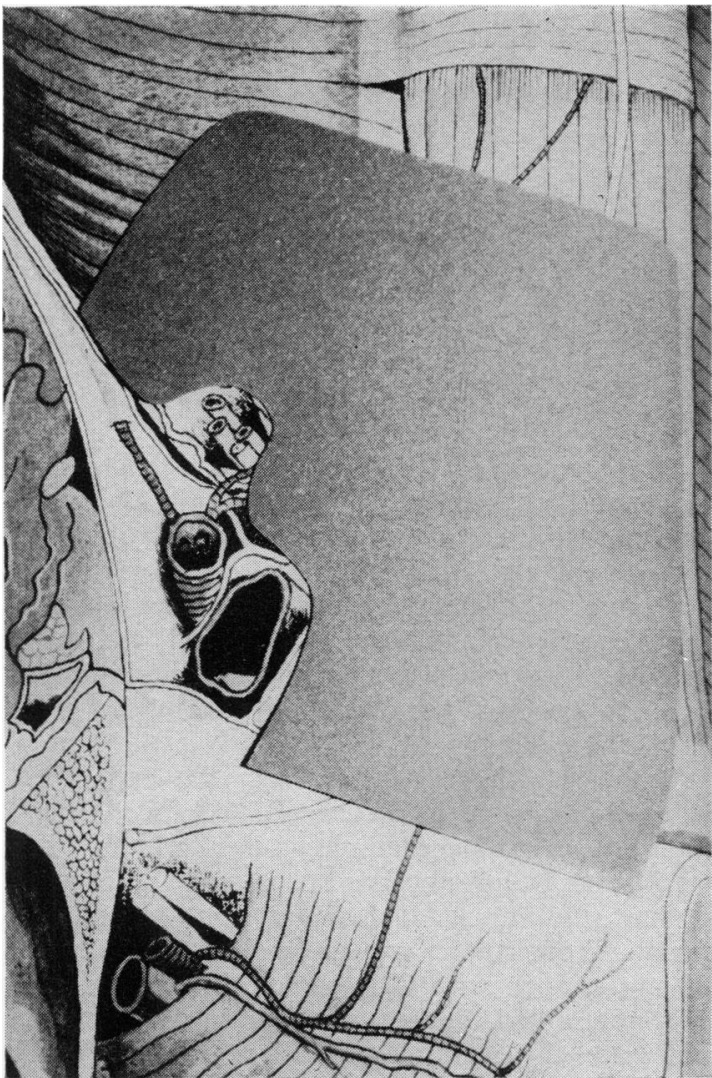

FIg. 4. The Marlex mesh prosthesis in position covers the posterior wall of the inguinal canal and femoral canal, and controls the internal inguinal ring. of the tissues or suture of structures under tension.

(8) Incipient hernia found at operation in the contralateral site can be dealt with prophylactically.

This paper is in the nature of a preliminary communication as complete evaluation of the operation will await the passage of time; however, I have been sufficiently impressed by the immediate and early post-operative results to feel justified in continuing its usage; long-term results will be reported in due course.

\section{Acknowledgments}

I would like to record my appreciation of $\mathrm{Mr}$ Henry Wapshaw, Consultant Surgeon, Western Infirmary, Glasgow, for his help and co-operation and of the Director and the staff of the Department of Medical Illustration, Cardiff Royal Infirmary for the photographic illustrations.

\section{References}

Henry, A.K. (1963) Operation for femoral hernia by a midline intra-peritoneal approach. Lancet, i, 230.

Moschcowitz, A.V. (1915) Hernia. Operative Therapeusis (Ed. by A. B. Johnson). Appleton, New York.

Nyhus, L.M. \& Harkins, H.N. (1964) Hernia. Pitman, London.

USHER, F.C. (1959) Further observation on the use of Marlex mesh: a new technique for the repair of inguinal hernia. Amer. Surg. 25, 792.

USHER, F.C. (1962) Hernia repair with Marlex mesh Surgery, 84, 325.

USHER, F.C. (1963) Hernia repair with knitted polypropylene mesh. Surg. Gynaec. Obstet. 117, 239.

USHER, F.C., OSChNER, J. \& TUTTLE, L.L.J. (1958) Use of Marlex mesh in the repair of incisional hernia. Amer. Surg. 24, 969. 